\title{
6-Phosphofructo-2-kinase/fructose-2,6-biphosphatase 4 acts as a protein kinase to regulate glioblastoma progression by activating the AKT/forkhead box 01 pathway
}

\author{
Kai Zhao'\#, Chaojun Yu², Ji Luo', Minhao Huang1', Qian Wen¹ and Ninghui Zhao'® \\ 1Neurosurgery Department, the Second Affiliated Hospital of Kunming Medical University, Kunming, Yunnan Province, China; 2 Neurosurgery \\ Department, 903 Hospital, Jiangyou City, Sichuan Province, China
}

\begin{abstract}
Abnormal expression of 6-phosphofructo-2-kinase/fructose-2,6-biphosphatase 4 (PFKFB4) is closely related to the occurrence and development of tumors, and PFKFB4 has been shown to function as a protein kinase. However, the molecular mechanisms through which PFKFB4 functions in glioblastoma (GBM) remain poorly understood. Accordingly, in this study, we assessed the roles of PFKFB4 in GBM. Compared to in adjacent tissues, PFKFB4 was highly expressed in GBM, and its expression level was negatively correlated with the overall survival time. In addition, knockdown of PFKFB4 inhibited the proliferation and invasion of GBM cells and promoted apoptosis. In a xenograft tumor model, tumor growth was inhibited by knockdown of PFKFB4 using short hairpin RNA. Further studies demonstrated that PFKFB4 is involved in regulating the AKT signaling pathway. Thus, PFKFB4 acts as a protein kinase to regulate GBM progression by activating the AKT/forkhead box 01 pathway, which may be a potential therapeutic target in GBM.
\end{abstract}

Keywords: PFKFB4, glioblastoma, protein kinase, AKT/FOXO1 pathway

Received: 16 July, 2021; revised: 29 September, 2021; accepted: 29 September, 2021; available on-line: 10 February, 2022

凶e-mail: zhaoninghui@hotmail.com

\#These authors contributed equally to this work

Acknowledgements of Financial Support: This study was supported by the National Natural Science Foundation of China (Grant No.: 81960459).

Abbreviations: GBM, glioblastoma multiforme; PFKFB4, fructose-2,6-biphosphatase 4; FOXO1, forkhead box O1; shRNA, short hairpin RNA; TCGA, The Cancer Genome Atlas; GO, Gene Ontology; KEGG, Kyoto Encyclopedia of Genes and Genomes

\section{INTRODUCTION}

Glioma has the highest incidence among all tumors of the central nervous system, and glioblastoma (GBM) is the most common type of glioma (Shen et al., 2021). Glioma is a neuroepithelial tumor with high malignancy, a high recurrence rate, and poor prognosis and often exhibits infiltrating rapid growth, proliferation, and strong invasion (Nasrollahzadeh et al., 2021). Despite advances in molecular diagnosis, surgical treatment, chemotherapy, and radiotherapy of glioma in recent years, the overall prognosis remains poor "'Correction to: Runx2 (RuntRelated Transcription Factor 2) Links the DNA Damage Response to Osteogenic Reprogramming and Apoptosis of Vascular Smooth Muscle Cells," 2021). The average 5 -year survival rate of patients with malignant glioma is only $4-29 \%$, and the average survival time is approxi- mately 1 year. Thus, glioma poses a major threat to human health (Kotowski et al., 2021).

6-Phosphofructo-2-kinase/fructose-2,6-biphosphatase 4 (PFKFB4) is a member of the PFKFB family and is widely present in various types of cells. Previous studies suggested that PFKFB4 is a bifunctional enzyme that acts as a phosphatase and kinase to mediate the formation and degradation of fructose-2,6-diphosphate, an allosteric activator of fructose phosphate kinase- 1 and the most effective stimulator of glycolysis to regulate cellular glycolysis levels (Yan et al., 2021; Zhang et al., 2021). In addition to its recognized roles in aerobic glycolysis, biosynthesis, and redox homeostasis, PFKFB4 is involved in a variety of biological processes, such as cell cycle regulation, autophagy, and transcriptional regulation $(\mathrm{Lu}$ et al., 2020; Wang et al., 2020). Moreover, PFKFB4 can function as a protein kinase. Indeed, a recent study revealed that PFKFB4 transfers a phosphate group from ATP to Ser857 on steroid receptor activation factor 3 (SRC-3) (Guo et al., 2020). SRC-3 is a member of the p160SRC family of nuclear receptor coactivators, and its overexpression has been detected in breast cancer, bladder cancer, glioma, and other solid tumors. Additionally, PFKFB4 was shown to control embryonic development and regulate AKT signaling by phosphorylating Ser473, independently of the glycolytic functions of PFKFB4 (Duan et al., 2020). Furthermore, the regulation of AKT signaling via PFKFB4-dependent phosphorylation is essential for premigratory and migratory neural crest formation, and deletion of PFKFB4 results in major defects in migration (Yi et al., 2019). PFKFB4 also promotes tumorigenesis and development in thyroid carcinoma; indeed, tumor growth, metastasis, and invasion were shown to be inhibited after knockout of PFKFB4. Mechanistically, PFKFB4 phosphorylation activates the AKT signaling pathway (Jiang et al., 2019). Taken together, these findings confirm that PFKFB4 acts as a protein kinase to activate tumor-promoting factors and mediate the occurrence and development of cancer.

Forkhead box O1 (FOXO1) is a widely expressed transcription factor of the FOXO family and an important tumor-suppressor gene that is downregulated in many types of tumor tissues (Kessler et al., 2019). FOXO1 is degraded after AKT-dependent phosphorylation, and a reduction in FOXO1 expression promotes tumor cell growth and proliferation, inhibits tumor cell apoptosis, enhances the invasion and metastasis of tumor cells, and stimulates blood vessel formation (Dasgupta et al., 2018; Jiang et al., 2018; Lim et al., 2018). However, it is unclear whether PFKFB4 participates in phosphorylation-mediated regulation of AKT/FOXO1 signaling as a protein kinase in glioma. 
Table 1. shRNA target sequences

\begin{tabular}{ll}
\hline shRNA & Sequence $\left(5^{\prime}-3^{\prime}\right)$ \\
\hline PFKFB4-shRNA1 & GATCCGCGCGGTGAACTTCAAAGGTCTCGAGACCTTGAAAGTTCACCGCGCTTाTा \\
\hline PFKFB4-shRNA2 & AATTAAAAAAGGCAGGAACTTCAGCAGAGATCTCGAGATCTCTGCTGAAGTTCCTGCCG \\
\hline PFKFB4-shRNA3 & GATCCGCTGGAGAGGCAAGAGAATGTCTCGAGACATTCTCTTGCCTCTCCAGCTTITT \\
\hline sh-control & GATCTGTTCTCCGAACGTGTCACGTTCAAGAGAACGTGACACGTTCGGAGAATTTTC \\
\hline
\end{tabular}

Note: shRNA = short hairpin RNA; PFKFB4 = fructose-2,6-biphosphatase 4

Accordingly, in this study, we performed RNA sequencing (RNA-Seq) to analyze the signaling pathways affected by knockdown of PFKFB4 in glioma cells. Our results suggest that PFKFB4 regulates AKT/FOXO1 signaling, providing insight into the mechanisms of PFKFB4 function in GBM.

\section{MATERIALS AND METHODS}

\section{Bioinformatics analysis}

PFKFB4 sequencing expression data were downloaded from The Cancer Genome Atlas (TCGA; https:// www.cancer.gov/about-nci/organization/ccg/research/ structural-genomics/tcga). Differential expression analysis was performed using the "EdgeR" package in R software (The R Project for Statistical Computing, Vienna, Austria). The survival and prognosis of patients with high and low expression of PFKFB4 were analyzed using the OncoLnc database (http://www.oncolnc.org/).

\section{Patients and samples}

Fresh GBM tissues and paracancerous tissues were collected during operation. Patients who had not undergone primary surgery and had not been administered radiotherapy or chemotherapy before the operation were included in the study. Their tissues were stored in liquid nitrogen tanks within $2 \mathrm{~h}$ of surgical resection. The Ethics Committee of the Second Affiliated Hospital of Kunming Medical University approved this study. Written informed consent was obtained from all donors or their close relatives before sample collection.

\section{Cell culture}

Human glioma cells (U87, U251, and T98G cells) and human cerebellar astrocytes (Hac cells) were cultured in Dulbecco's modified Eagle medium (Hyclone, Logan, UT, USA). U87 cells stably expressing short hairpin RNA (shRNA) targeting PFKFB4 (sh-PFKFB4), short hairpin control (sh-control), cells overexpressing PFKFB4 (OE-PFKFB4), and overexpression control (OE-control) cells were grown in minimum essential medium (MEM; Biosharp, Beijing, China) supplemented with $10 \%$ fetal bovine serum (Gibco, Grand Island, NY, USA) and $1 \%$ penicillin/streptomycin (Gibco) in an incubator at $37^{\circ} \mathrm{C}$ with $5 \% \mathrm{CO}_{2}$.

\section{Stable transfection of U87 cells with sh-PFKFB4 and OE-PFKFB4}

Four shRNAs were cloned into the lentiviral vector pGMLV-SC5-puro using a high-purity plasmid cassette (DP107; Tiangen Biotech, Beijing, China) and KOD-Plus-Neo DNA polymerase (Toyobo, Osaka, Japan). The sequencing results of the PFKFB4 interference vector and negative control are shown in Table 1. pGMLV-SC5-puro was used as a negative control. The constructed lentivirus vector and auxiliary packaging vector plasmid were cotransfected into 293T cells with HG transgene reagent (Qiagen, Hilden, Germany). After collecting the virus-rich supernatant, U87 cells were infected with the packaged lentivirus and lentivirus negative control, and stable cells were selected with puromycin. Infected cells showing green fluorescence were detected by flow cytometry, and the infection efficiency was evaluated. Quantitative reverse transcription polymerase chain reaction (qRT-PCR) and western blotting were performed to detect the best interference effect of shRNA. Similarly, 293T cells were cotransfected with the constructed lentiviral vector and packaging mix, and the titer of lentivirus solution (15682PGMLV-CMVH_PFKFB4-EF1-ZsGreen1-T2A-Puro) was determined. U $\overline{8} 7$ cells were infected with packaged lentivirus and lentivirus negative control, and stable strains were obtained. Infected cells expressing green fluorescence were detected by flow cytometry, and the infection efficiency was evaluated. qPCR and western blotting were conducted to verify the effects of transfection.

\section{qRT-PCR}

Primers designed using DNAMAN were synthesized by Sangon Biotech (Shanghai, China) and are shown in Table 2. RNAiso Plus (Takara, Shiga, Japan) was added to the cells to extract total RNA. A RevertAid First Strand cDNA Synthesis kit (Thermo Fisher Scientific, Waltham, MA, USA) was used to synthesize cDNA. qRT-PCR was conducted for 40 cycles utilizing FastStart Universal SYBR Green Master Mix with ROX (Roche, Basel, Switzerland) with 7500 Real-Time PCR system (Applied Biosystems, Foster City, CA, USA). The expression level was normalized to that of the internal reference GAPDH, and the relative expression level was determined using the $\Delta \Delta \mathrm{CT}$ method.

\section{Western blotting}

The following antibodies were used for western blotting: anti-PFKFB4 (1:3000, rabbit; Abcam, Cambridge,

Table 2. PCR primers

\begin{tabular}{lll}
\hline Gene & Forward primer sequence & Reverse primer sequence \\
\hline PFKFB4 & GTGATGAGGCTACGGAGGACTTC & GTAATATACGATGCGGCTCTGG \\
\hline GAPDH & GTCTCCTCTGACTTCAACAGCG & ACCACCCTGTTGCTGTAGCCAA \\
\hline
\end{tabular}


UK), anti-Bax (1:5000, rabbit; Proteintech, Rosemont, IL, USA), anti-phospho-AKT (Ser473) (1:2000, mouse; Proteintech), anti-AKT (1:1000, rabbit; Proteintech), antiFOXO1 (1:1000, rabbit; Cell Signaling Technology, Danvers, MA, USA), and anti- $\beta$-actin (1:1000, rabbit; Cell Signaling Technology). Standard procedures were used to perform western blotting. ImageJ software (v1.5.2, National Institutes of Health, Bethesda, MD, USA) was used to perform densitometry analysis of the bands.

\section{Cell proliferation assay}

The cells (sh-control and sh-PFKFB4; OE-control and OE-PFKFB4) were seeded into 96-well plates $(2,500$ cells/well, five plates), and the results were recorded for 4 days. After every $24 \mathrm{~h}$, the cells were incubated with Cell Counting Kit- 8 reagent (Beyotime, Shanghai, China) for $4 \mathrm{~h}$, and the absorbance of each well was detected at a wavelength of $450 \mathrm{~nm}$ by spectrophotometry. The final optical density value was determined using the following equation: final value $=$ measured absorbance - blank well absorbance.

\section{EdU staining assay}

BeyoClick EdU-555 (Beyotime) was used to detect cell proliferation. We inoculated $4 \times 10^{5}$ cells (sh-PFKFB4, sh-control) into 6-well culture plates. EdU-labeled cells were fixed with $4 \%$ paraformaldehyde and permeabilized with $1 \mathrm{~mL} 0.3 \%$ Triton X-100 solution, followed by staining with Hoechst 33342. EdU staining was detected using an inverted fluorescence microscope (20x).

\section{Wound healing assays}

The cells (sh-control and sh-PFKFB4) were inoculated into 6-well culture plates, and two horizontal lines were drawn on the bottom of the outer surface of the plate. The cells were grown in MEM containing 10\% fetal bovine serum until reaching confluence. A sterilized $10-\mu \mathrm{L}$ pipette tip was then used to scratch the surface of the cell layer. After washing gently with phosphate-buffered saline, the cells were cultured in serum-free MEM. Cell growth across the scratch was visualized using a brightfield microscope at $24-\mathrm{h}$ intervals. The endpoint was considered as the time at which the cells had grown across the scratch. The area of the scratch covered by the cells at each time point was determined using ImageJ software.

\section{Transwell assays}

Cell migration was evaluated using Transwell cells (pore size: $8.0 \mu \mathrm{m}$; COSTAR, Corning, Inc., Corning, NY, USA). The cells (sh-control and sh-PFKFB4) were added to the upper chamber at a density of $3 \times 10^{4}$ cells in $200 \mu \mathrm{L}$ serum-free medium. After culturing for $72 \mathrm{~h}$, the cells were fixed in 4\% paraformaldehyde for $30 \mathrm{~min}$ and then stained with crystal violet. Cotton swabs were used to remove cells that had not migrated through the Transwell inserts, and stained cells that had migrated through the membrane were visualized using a microscope.

\section{RNA-Seq}

A Total RNA Extractor (TRIzol) kit (cat. no. B511311; Sangon Biotech) was used to extract total RNA from the cells (stable shPFKFB4- and sh-control-transfected U87 cells; triplicate samples were prepared). A total of $2 \mu \mathrm{g}$ RNA per sample was used as input material for RNA sample preparations. Sequenc- ing libraries were generated using a Hieff NGS MaxUp Dual-mode mRNA Library Prep Kit for Illumina (cat. no. 12301ES96; Yeasen, Shanghai, China) following the manufacturer's recommendations. To select cDNA fragments that were 150-200 base pairs in length, the library fragments were purified with Hieff NGS DNA Selection Beads DNA (cat. no. 12601ES56; Yeasan). PCR was performed with $2 \times$ Super Canace High-Fidelity Mix, Primer mix, and Adapter-Ligated DNA. Finally, the PCR products were purified using Hieff NGS DNA Selection Beads, and the library quality was assessed on a Qubit 2.0 Fluorometer (Thermo Fisher Scientific). The libraries were quantified and pooled. Paired-end sequencing of the libraries was performed on a HiSeq NovaSeq 6000 sequencer (Illumina, San Diego, CA, USA). The DESeq2 package was used for differential gene expression analysis $\left(\mid \log _{2}\right.$ [fold-change] $\left.>1 \mid, P<0.05\right)$, and Gene Ontology and Kyoto Encyclopedia of Genes and Genomes enrichment analyses of differential expression genes were performed to evaluate biological functions.

\section{Flow cytometry}

Annexin Vdome 633 (lot no. NY601; Dojindo, Kumamoto, Japan) was used to detect apoptotic cells (sh-control and sh-PFKFB4; OE-control and OE-PFKFB4) according to the manufacturer's instructions. Flow cytometry was performed on a BD FACS Celesta multicolor cell analyzer (BD Biosciences, Franklin Lakes, NJ, USA). FlowJo-V10 software (TreeStar, Ashland, OR, USA) was used to analyze the data.

\section{Caspase-3 activity assay}

A Caspase-3 Activity Assay Kit (Beyotime) was used to detect caspase-3 activity in sh-control and sh-PFKFB4 cells. A microplate spectrophotometer was used to quantify caspase- 3 activity by measuring the absorbance at $405 \mathrm{~nm}$. Caspase-3 activity was expressed as the foldchange in enzyme activity compared with that of the control.

\section{Immune-deficient mouse experiments}

This experiment complied with ARRIVE guidelines, the U.K. Animals (Scientific Procedures) Act, 1986 and associated guidelines, and EU Directive 2010/63/EU. Six immune-deficient male mice, each weighing 18-22 g (6 weeks old), were purchased from the animal room of Kunming Medical University (Yunnan, China). Mice were maintained in a specific pathogen-free facility and randomly assigned to two groups (three mice per group). Tumor cells $\left(1.2 \times 10^{6}\right.$ sh-PFKFB4 or sh-control cells) were subcutaneously injected into the left armpit skin $(0.2 \mathrm{~mL})$ over an injection time of approximately $1 \mathrm{~min}$. After 4 weeks, the tumors were dissected, their sizes and weights were measured, and western blotting was performed to evaluate PFKFB4 expression.

\section{Ethics committee approval}

This study was approved by the Ethics Committee of the Second Affiliated Hospital of Kunming Medical University. Under committee supervision, samples were used after obtaining written informed consent from the donor or next of kin.

\section{Statistics}

All experiments were repeated three times independently. GraphPad Prism 8.0 (GraphPad, Inc., La Jolla, 


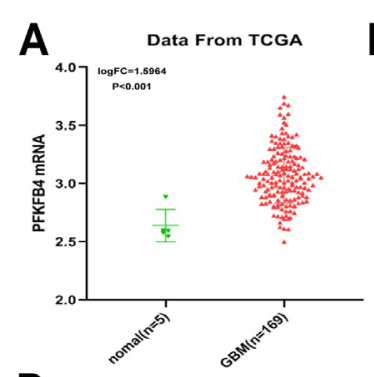

D

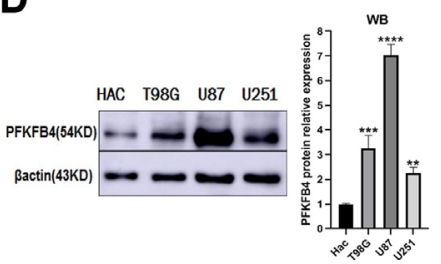

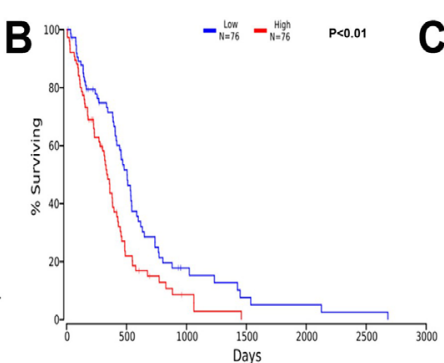

E

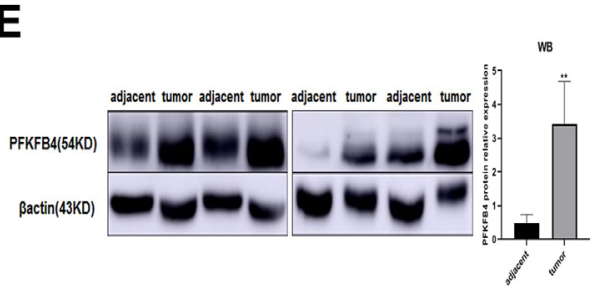

Figure 1. PFKFB4 was highly expressed in glioblastoma (GBM) and may be associated with poor patient prognosis.

(A) Expression of PFKFB4 in normal brain tissue $(n=5)$ and GBM tissue $(n=169)$ using data from The Cancer Genome Atlas database. ${ }^{* * *} P<0.001$. (B) Effect of high and low expression of PFKFB4 on the survival of patients with GBM in the OncoLnc database. ${ }^{* *} P<0.01$. (C, D) qPCR and western blotting analysis of PFKFB4 expression in GBM and normal tissues. ${ }^{* * *} P<0.001,{ }^{* *} P<0.01,{ }^{*} P<0.05$. (E) GBM and adjacent tissues were collected as clinical samples. Western blotting was used to evaluate PFKFB4 expressio. ${ }^{* *} P<0.01$.

CA, USA) was used to produce graphs. SPSS 21.0 software (SPSS, Inc., Chicago, IL, USA) was used for statistical analysis of the data. Measurement data were presented as the means \pm standard deviations, and differences between the two groups were evaluated using $t$-tests. Results with $P$ values less than 0.05 were considered statistically significant.

\section{RESULTS}

\section{PFKFB4 was upregulated and correlated with poor survival in GBM}

Based on TCGA data, PFKFB4 levels in the tumor tissues were significantly higher than those in normal tissues $(P<0.001$; Fig. 1A). To evaluate the clinical importance of PFKFB4, patients were divided into high and low expression groups according to the median risk score of PFKFB4. According to the OncoLnc database, patients with high expression of PFKFB4 typically had shorter survival times $(P<0.01$; Fig. 1B). These data suggest that PFKFB4 is a poor prognostic factor for GBM.

The mRNA (Fig. 1C) and protein levels of PFKFB4 (Fig. 1D) in GBM cells were higher than those in normal astrocytes $(P<0.05)$, particularly in U87 cells. In fresh surgical samples, western blotting showed that PFKFB4 average expression was significantly higher in cancer tissues than in adjacent tissues $(P<0.01$; Fig. 1E).
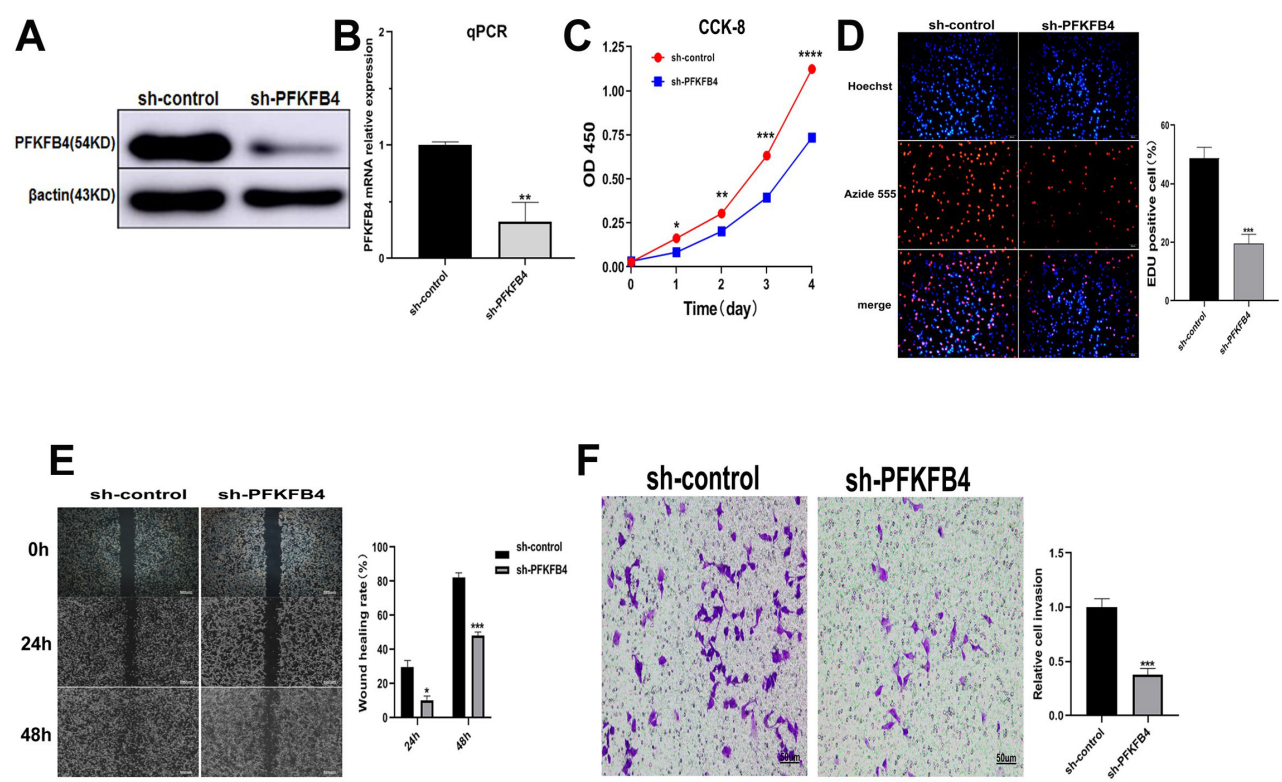

sh-PFKFB4

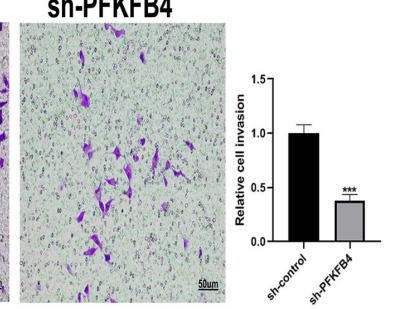

Figure 2. Knockdown of PFKFB4 inhibited the proliferation, migration, and invasion of glioblastoma (GBM).

(A, B) Western blotting and GPCR were used to evaluate the expression of PFKFB4 in sh-PFKFB4 and control cells. ${ }^{* *} P<0.01$. (C) CCK-8 assays were used to evaluate the effects of PFKFB4 interference on the proliferation of U87 cells. ${ }^{* * * * *} P<0.0001,{ }^{* * *} P<0.001,{ }^{* *} P<0.01$, ${ }^{*} P<0.05$. (D) Effects of PFKFB4 interference on the percentages of EDU-positive U87 cells. ${ }^{* * *} P<0.001$. (E, F) Wound healing (E) and transwell (F) assays were used to evaluate the effects of PFKFB4 knockdown on the migration and invasion of U87 cells. ${ }^{* * *} P<0.001,{ }^{*} P<0.05$. 

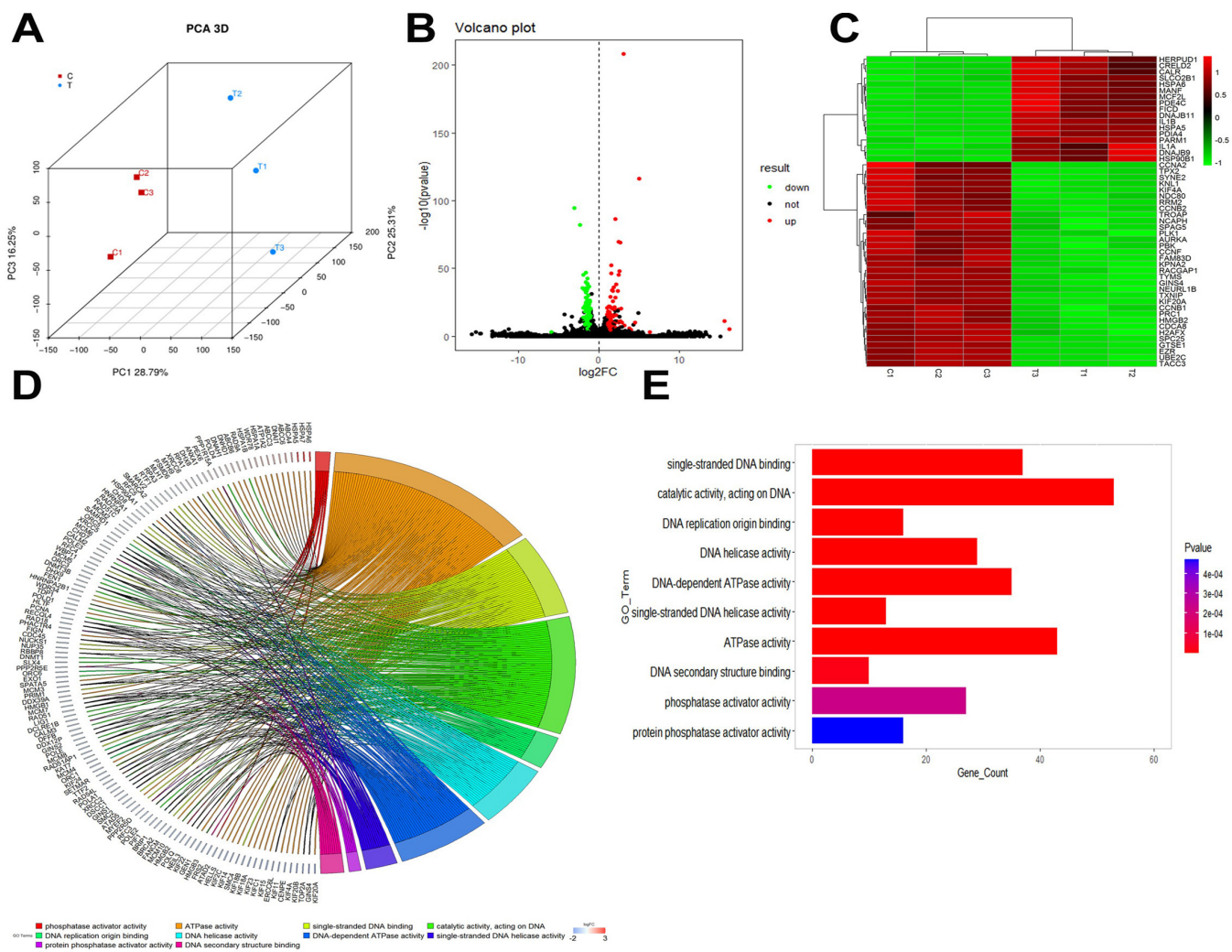

$\mathbf{F}$

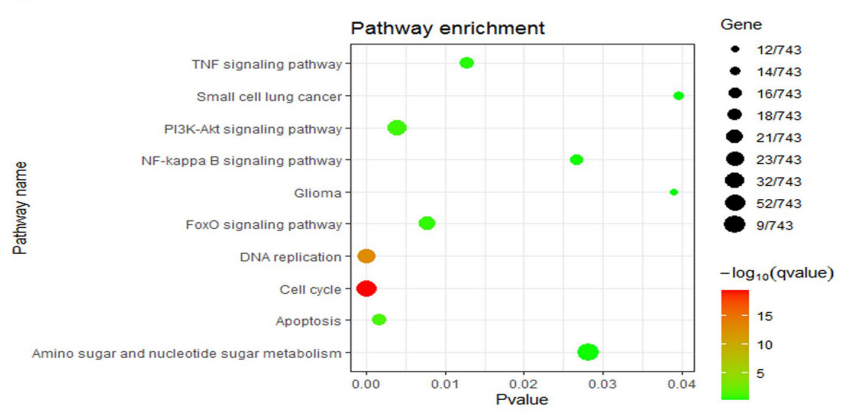

H
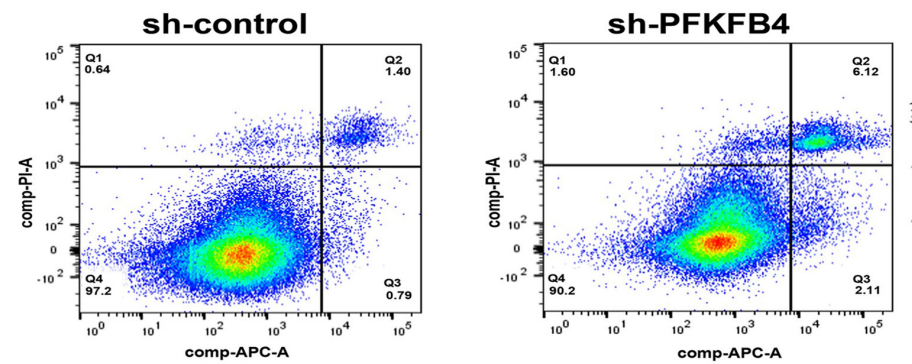
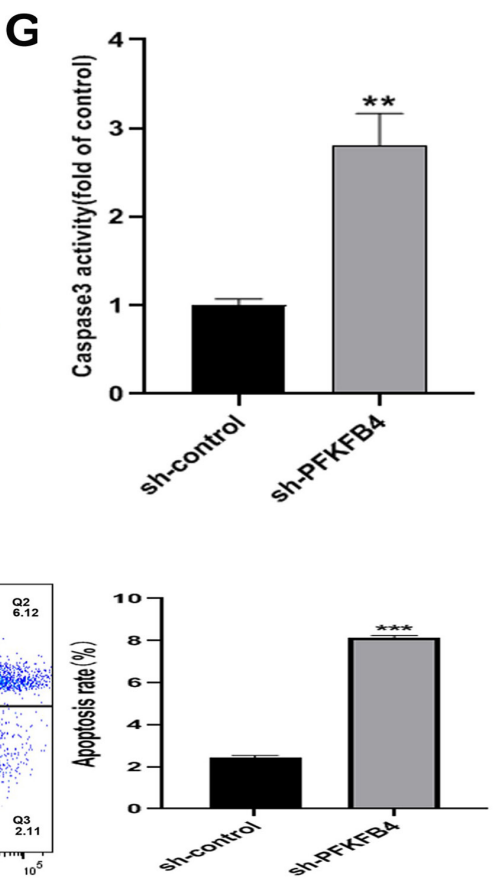

Figure 3. RNA-Seq analysis demonstrated that knockdown of PFKFB4 affected protein kinase activity, DNA repair, and apoptosis.

(A) Principal component analysis diagram. The red square represents the group of sh-control U87 cells, and the blue circle represents the sh-PFKFB4 group. The scale of the horizontal and longitudinal coordinates is the relative distance. The two groups of cells were classified clearly. (B) Differential analysis of three sh-PFKFB4 and three sh-control stable U87 cell lines revealed 359 differentially expressed genes, with 196 downregulated (green) and 163 upregulated (red) genes. (C) Heatmaps showing the correlations of PFKFB4 with the top 50 gene expression signatures in RNA-Seq analysis. Rows represent genes, and columns represent samples. (D, E) Gene Ontology (GO) enrichment analyses of differentially expressed genes from RNA-Seq of U87 cells with PFKFB4 knockdown. In the GO Chord plot (D), differentially expressed genes are shown on the left, different GO terms are on the right, and the colored ribbon connected to the genes represents enrichment of the gene in this GO term. In the bar plot (E), the left side represents the GO terms, the horizontal axis represents the gene counts, and color represents the $P$ value. (F) Kyoto Encyclopedia of Genes and Genomes enrichment analyses of differentially expressed genes based on RNA-Seq of U87 cells with PFKFB4 knockdown. The left side of the dot plot shows the pathway names, and the right side shows the gene ratio. The horizontal axis represents the $P$ value. (G) Effects of PFKFB4 knockdown on caspase-3 activity. ${ }^{* *} P<0.01$. (H) Effects of PFKFB4 knockdown on apoptosis rates in U87 cells. ${ }^{* * *} P<0.001$. 

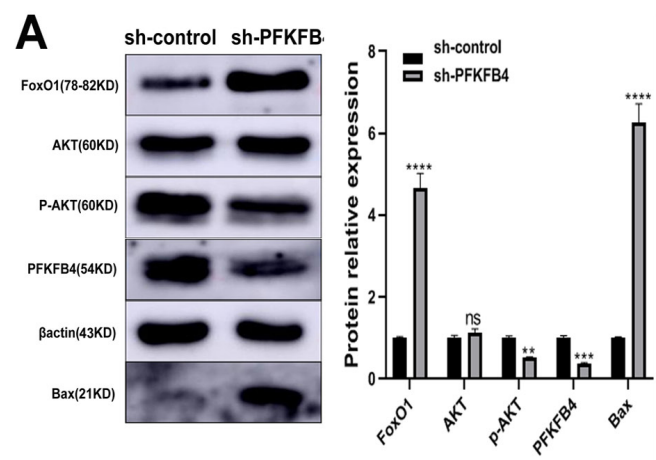

B

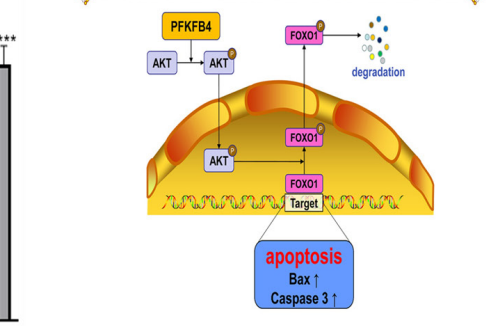

C
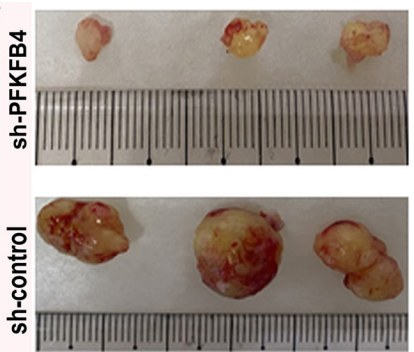

E

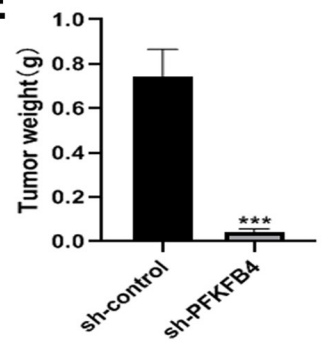

$\mathbf{F}$
WB

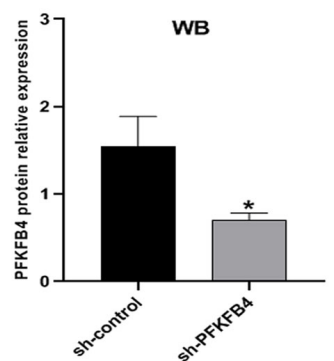

Figure 4. PFKFB4/AKT/FOXO1 pathway regulated glioblastoma progression.

(A) Western blot analysis of the effects of PFKFB4 knockdown on phopsho-AKT, total AKT, FOXO1, and Bax protein levels. ${ }^{* *} P<0.01$. (B) Mechanism of the PFKFB4/AKT/FOXO1 pathway in GBM. FOXO1 inhibits apoptosis by increasing downstream caspase-3 activity and Bax expression in the nucleus. When PFKFB4 phosphorylation activates AKT, the latter enters the cell nucleus, resulting in FOXO1 phosphorylation and translocation from the nucleus to the cytoplasm for degradation. (C, D) Animal experiments showing the effects of PFKFB4 knockdown on tumor volume (C) and weight (D). ${ }^{* * *} P<0.001$. (E) Western blotting of PFKFB4 expression in tumor tissues from the xenograft model. ${ }^{*} P<0.05$.

\section{PFKFB4 promoted growth and migration in GBM cells}

Stable-knockdown PFKFB4 cell lines were constructed using lentivirus. The best interference effect was detected using shRNA3 (Supplemental Fig. 1A, B at https://ojs.ptbioch.edu.pl/index.php/abp/). Therefore, the PFKFB4-shRNA3 U87 cell line was selected for followup studies. Western blotting and qRT-PCR confirmed that PFKFB4 was effectively knocked down (Fig. 2A, B). Compared with the control cell line, U87 cells transfected with stable sh-PFKFB4 showed growth inhibition (Fig. 2C, D). Moreover, wound healing and Transwell assays showed that migration and invasion were significantly inhibited after knockdown of PFKFB4 (Fig. 2E, F). We also established stable PFKFB4-overexpressing U87 glioma cells, which was confirmed through western blotting and qPCR analyses (Supplemental Fig. 2A, B). Compared with the control cell line, PFKFB4-overexpressing cells showed no obvious changes in survival or apoptosis (Supplemental Fig. 2C, D at https://ojs.ptbioch.edu.pl/index.php/abp/).

\section{Knockdown of PFKFB4 affected protein phosphokinase activity, DNA repair, and apoptosis}

To further explore the roles of PFKFB4 in GBM, RNA-Seq analysis was performed in U87 cells stably transfected with sh-PFKFB4 or sh-control. The principal component analysis results of the two groups showed similarities between the cell lines (Fig. 3A). In PFKFB4knockdown cells, we identified 359 differentially expressed genes, with 196 downregulated and 163 upregulated genes (Fig. 3B), which were visualized in a heat map (Fig. 3C). According to the Gene Ontology (GO) annotations, PFKFB4 was significantly associated with protein phosphatase activator activity, DNA replication, and ATPase activity (Fig. 3D, E). Additionally, Kyoto
Encyclopedia of Genes and Genomes (KEGG) analysis of the RNA-Seq results indicated that different genes were enriched in the phosphatidylinositol 3-kinase/AKT, $\mathrm{FOXO}$, and tumor necrosis factor signaling pathways (Fig. 3F). As verification of these activities, we observed that knockdown of PFKFB4 enhanced caspase-3 activity and apoptosis rates (Fig. 3G, H), suggesting that PFKFB4 inhibited apoptosis in GBM.

\section{PFKFB4-dependent AKT signaling was essential for glioma development}

Western blotting showed that phospho-AKT levels were decreased after inhibition of PFKFB4, whereas there were no significant differences in the expression of total AKT (Fig. 4A). During the occurrence and development of glioma, PFKFB4 phosphorylation may activate $\mathrm{AKT}$, which then enters the nucleus, leading to activation of FOXO1 phosphorylation; FOXO1 is then transferred from the nucleus to the cytoplasm, where it is degraded, thereby inhibiting apoptosis and promoting proliferation in cancer cells. When PFKFB4 was inhibited, FOXO1 accumulated in the nucleus, leading to increased Bax and caspase-3 expression (Fig. 4B). This result is consistent with the findings of RNA-seq analysis. Thus, the PFKFB4/AKT/FOXO1 signaling pathway may be important in the occurrence and development of GBM.

Finally, to explore the tumor-promoting effects of PFFKB4 in vivo, we established an animal model by xenotransplantation. The tumor volume and body weights of mice were significantly lower in the shPFKFB4 group than the control group $(P<0.001$; Fig. 4C, D), consistent with our in vitro experimental results. Moreover, PFKFB4 average expression was low in transplanted tumors derived from shPFKFB4 cells $(P<0.05$; Fig. 4E). 


\section{DISCUSSION}

PFKFB4 regulates the concentration of the intracellular allosteric glycolysis activator fructose-2,6-diphosphate (Gao et al., 2018; Ostrom et al., 2017). Recently, high expression of PFKFB4 was observed in many tumors, suggesting that this protein has an important role in the initiation and progression of cancer (Figueiredo et al., 2017; Pegoraro et al., 2015; Pranckeviciene \& Bunevicius, 2015). Additionally, high PFKFB4 expression is associated with a shorter overall survival time and more frequent metastasis (Strohecker et al., 2015; Yi et al., 2019; Zhao et al., 2015). Many reports have also described the role of PFKFB4 in brain tumors. For example, downregulation of PFKFB4 leads to decreased lactic acid levels and ATP production in brain cancer stem cells, thereby inducing apoptosis (Minchenko et al., 2014). Additionally, the PFKFB3/PFKFB4 mRNA ratio in isocitrate dehydrogenase wild-type glioblastoma $(7.7: 1)$ is lower than that in isocitrate dehydrogenase mutant low-grade astrocytoma (36.5:1), suggesting that the ratio is related to the degree of malignancy, patients with a higher PFKFB3/ PFKFB4 mRNA ratio (14 months) show a significantly longer overall survival time than patients with a lower ratio (9 months) (Omuro \& DeAngelis, 2013).

RNA-Seq can be used to study differences in gene expression at the whole-genome level, enabling accurate quantification, high repeatability, a wide detection range, and reliable analyses. This new approach has revolutionized systematic analyses of pathways and has gradually become a leading experimental technology in the study of cancer biology. Thus, RNA-Seq is expected to yield new targets for tumor gene therapy, facilitating the accurate detection of cancer. In our study, RNA-seq showed that in the shPFKFB4 group, the expression of xanthine dehydrogenase, adenosine monophosphate deaminase 1 , and 6-phosphofructokinase 1 genes were downregulated, thus inhibiting glycolysis and the pentose phosphate pathway, whereas the expression of FOXO1 and Bax was upregulated. The annotation of GO terms showed that the regulation of PFKFB4 may affect DNA replication and DNA binding, which is closely related to tumor proliferation and apoptosis (Yun et al., 2012). PFKFB4 plays an important role in the Warburg effect of various malignant tumor cells and can promote glycolysis and the pentose phosphate pathway ( $\mathrm{Lu}$ et al., 2020; Yan et al., 2021), which enables cancer cells to synthesize DNA in a microenvironment with a limited oxygen supply. Furthermore, cancer cells continue to proliferate while producing lactic acid to acidify the adherent tissue, thus accelerating metastasis. The pentose phosphate pathway directly participates in the production of nicotinamide adenine dinucleotide and the synthesis of nucleic acid and DNA, which lead to the growth of cancer cells (Goidts et al., 2012). knockdown PFKFB4 in prostate cancer cells can induce their apoptosis and Inhibit the growth of tumors in vivo (Ros et al., 2012). RNA-seq showed that PFKFB4 is involved in the phosphorylation activity, and combined with the results of the KEGG pathway enrichment analysis, it showed that PFKFB4 activated the PI3K/AKT and FOXO signaling pathways (Fig. 3F). Western blotting confirmed that shPFKFB4 could significantly inhibit the activation of AKT phosphorylation and that the expression of the tumor suppressor FOXO1 increased in shPFKFB4 cells (Fig. 4A). The regulatory effect of PFKFB4 on AKT has been reported (Duan et al., 2020; Yi et al., 2019). however, there are only a few studies on this regulatory mechanism in tumors, and ours is one such study. The PFKFB4/
AKT/FOXO1 pathway plays an important regulatory role in the occurrence and development of gliomas.

Many studies have focused on the roles of PFKFB4 in tumors. PFKFB4 is a key multifunctional regulatory factor with important roles in tumor metabolism proliferation, metastasis, autophagy, and apoptosis. PFKFB4 can enhance the migration/invasion ability of breast cancer cells, both in vitro and in vivo, by inducing hyaluronan synthase 2 expression and hyaluronan production (Strohecker et al., 2015). In addition, PFKFB4 can be used as a molecular fulcrum to combine glycolysis with autophagy and can be a promising target for tumor therapy. PFKFB4 knockout in prostate cancer cells increased p62 and reactive oxygen species levels and autophagy flux, thus affecting the growth of cancer cells (Zhang et al., 2011). Recently, a study reported downregulation of PFKFB4 inhibits the SRC3/AKT/mammalian target of rapamycin pathway, resulting in autophagy to promote tumor cell apoptosis (Jeon et al., 2011). Bruceine A, a quassinoid isolated from dried fruits, can inhibit cell cycle arrest and apoptosis mediated by the PFKFB4/ glycogen synthase kinase $3 \beta$ pathway in pancreatic cancer (Yalcin et al., 2009). Additionally, PFKFB4 is involved in regulating apoptosis in melanoma and hepatocellular carcinoma cells (Minchenko et al., 2005b; Valera et al., 2007). Based on previous studies and our RNA-Seq results, we focused on the regulatory relationships between PFKFB4 and AKT. Our results showed that PFKFB4 activates the AKT signaling pathway in glioma cells, consistent with previous studies of embryonic development (Duan et al., 2020). Moreover, FOXO1 has been shown to exert antitumor effects in many cancers, including antiproliferative and pro-apoptotic effects (Kessler et al., 2019). Decreased AKT activity and FOXO1 phosphorylation result in the suppression of FOXO1 transfer from the nucleus to the cytoplasm, thereby blocking degradation and increasing nuclear accumulation of FOXO1 (Minchenko et al., 2005a), resulting in increased caspase-3 and Bax expression (Pilkis et al., 1995) which induces apoptosis in glioma cells. Our study suggests that PFKFB4/AKT/FOXO1 signaling regulates glioma development and progression.

Regulation of AKT by PFKFB4 may be involved in drug resistance in glioma. Temozolomide is still the only first-line anti-glioma chemotherapeutic drug available on the market. This drug can be administered orally and can cross the blood-brain barrier; however, temozolomide also activates the AKT pathway and can cause insensitivity or resistance in glioma cells. Some studies showed that combination treatment with the inhibitor Y294002 with temozolomide can significantly increase apoptosis rates and reduce cell invasiveness via inhibition of AKT phosphorylation and Bcl-2 expression, thereby increasing cytotoxicity in tumors (Rousseau \& Hue, 1993). Thus, in future studies, we aim to evaluate the combined effects of the PFKFB4 inhibitor 5-(n-(8-methoxy-4-quinolyl) amino)pentyl nitrate and temozolomide in the treatment of glioma.

Overall, our findings provide important insight into the rarely studied roles of PFKFB4 in glioma. We confirmed that PFKFB4 functions as a protein kinase to regulate GBM progression by activating the AKT/FOXO1 pathway. Additionally, knockdown of PFKFB4 blocked the phosphorylation of AKT, resulting in increased FOXO1 and Bax expression, enhanced caspase-3 activity, decreased cell proliferation and migration ability, and increased apoptosis in cancer cells. Taken together, these findings suggest that PFKFB4 is a promising therapeutic target for treating glioma. 


\section{Declarations of interest}

None.

\section{REFERENCES}

Correction to: Runx2 (Runt-Related Transcription Factor 2) Links the DNA Damage Response to Osteogenic Reprogramming and Apoptosis of Vascular Smooth Muscle Cells (2021). Arterioscler Thromb Vasc Biol 41: e497. https://doi.org/10.1161/ATV.0000000000000146

Dasgupta S, Rajapakshe K, Zhu B, Nikolai BC, Yi P, Putluri N, Choi JM, Jung SY, Coarfa C, Westbrook TF, Zhang XH, Foulds CE, Tsai SY, Tsai MJ, O’Malley BW (2018) Metabolic enzyme PFKFB4 activates transcriptional coactivator SRC-3 to drive breast cancer. Nature 556: 249-254. https://doi.org/10.1038/s41586-018-0018-1

Duan R, Liang X, Chai B, Zhou Y, Du H, Suo Y, Chen Z, Li Q, Huang X (2020) xIsorhamnetin Induces Melanoma Cell Apoptosis via the PI3K/Akt and NF-B Pathways. Biomed Res Int 2020: 1057943, https://doi.org/10.1155/2020/1057943

Figueiredo AL, Maczkowiak F, Borday C, Pla P, Sittewelle M, Pegoraro C, Monsoro-Burq AH (2017) PFKFB4 control of AKT signaling is essential for premigratory and migratory neural crest formation. Development (Cambridge, England) 144: 4183-4194. https://doi. org/10.1242/dev.157644

Gao R, Liu Y, Li D, Xun J, Zhou W, Wang P, Liu C, Li X, Shen W, Su W, Qiao H, Stupack DG, Luo N (2018) PFKFB4 Promotes Breast Cancer Metastasis via Induction of Hyaluronan Production in a p38-Dependent Manner. Cell Physiol Biochem 50: 2108-2123. https://doi.org/10.1159/000495055

Goidts V, Bageritz J, Puccio L, Nakata S, Zapatka M, Barbus S, Toedt G, Campos B, Korshunov A, Momma S, Van Schaftingen E, Reifenberger G, Herold-Mende C, Lichter P, Radlwimmer B (2012) RNAi screening in glioma stem-like cells identifies PFKFB4 as a key molecule important for cancer cell survival. Oncogene 31: 32353243. https://doi.org/10.1038/onc.2011.490

Guo Q, Chen Q, Zhang Y, Zhou W, Li X, Li C, Zhang Y, Chen H, Liu P, Chu Y, Sun T, Jiang C (2020) Click-nucleic-acid-containing codelivery system inducing collapse of cellular homeostasis for tumor therapy through bidirectional regulation of autophagy and glycolysis. ACS Appl Mater Interfaces 12: 57757-57767. https://doi. org/10.1021/acsami.0c18361

Jeon YK, Yoo DR, Jang YH, Jang SY, Nam MJ (2011) Sulforaphane induces apoptosis in human hepatic cancer cells through inhibition of 6-phosphofructo-2-kinase/fructose-2,6-biphosphatase4, mediated by hypoxia inducible factor-1-dependent pathway. Biochim Biophys Acta 1814: 1340-1348. https://doi.org/10.1016/j.bbapap.2011.05.015

Jiang M, Chen Y, Deng L, Luo X, Wang L, Liu L (2019) SPAG6Upregulation of in Myelodysplastic Syndrome: Knockdown Inhibits Cell Proliferation via AKT/FOXO Signaling Pathway. DNA Cell Biol 38: 476-484. https://doi.org/10.1089/dna.2018.4521

Jiang S, Li T, Yang Z, Hu W, Yang Y (2018) Deciphering the roles of FOXO1 in human neoplasms. Int J Cancer 143: 1560-1568. https:// doi.org/10.1002/ijc.31338

Kessler R, Fleischer M, Springsguth C, Bigl M, Warnke JP, Eschrich K (2019) Prognostic value of PFKFB3 to PFKFB4 mRNA ratio in patients with primary glioblastoma (IDH-Wildtype). I Neuropathol Exp Neurol 78: 865-870. https://doi.org/10.1093/jnen/nlz067

Kotowski K, Rosik J, Machaj F, Supplitt S, Wiczew D, Jabłońska K, Wiechec E, Ghavami S, Dziegiel P (2021) Role of PFKFB3 and PFKFB4 in cancer: genetic basis, impact on disease development/ progression, and potential as therapeutic targets. Cancers (Basel) 13. https://doi.org/10.3390/cancers13040909

Lim M, Xia Y, Bettegowda C, Weller M (2018) Current state of immunotherapy for glioblastoma. Nat Rev Clin Oncol 15: 422-442. https:// doi.org/10.1038/s41571-018-0003-5

Lu H, Chen S, You Z, Xie C, Huang S, Hu X (2020) PFKFB4 negatively regulated the expression of histone acetyltransferase GCN5 to mediate the tumorigenesis of thyroid cancer. Dev Growth Differ 62: 129-138. https://doi.org/10.1111/dgd.12645

Minchenko OH, Ochiai A, Opentanova IL, Ogura T, Minchenko DO, Caro J, Komisarenko SV, Esumi H (2005a) Overexpression of 6-phosphofructo-2-kinase/fructose-2,6-bisphosphatase-4 in the human breast and colon malignant tumors. Biochimie 87: 1005-1010. https://doi.org/10.1016/j.biochi.2005.04.007

Minchenko OH, Opentanova IL, Ogura T, Minchenko DO, Komisarenko SV, Caro J, Esumi H (2005b) Expression and hypoxia-responsiveness of 6-phosphofructo-2-kinase/fructose-2,6-bisphosphatase 4 in mammary gland malignant cell lines. Acta Biochim Pol 52: $881-888$

Minchenko OH, Tsuchihara K, Minchenko DO, Bikfalvi A, Esumi H (2014) Mechanisms of regulation of PFKFB expression in pancreatic and gastric cancer cells. World J Gastroenterol 20: 13705-13717. https://doi.org/10.3748/wjg.v20.i38.13705
Nasrollahzadeh A, Momeny M, Fasehee H, Yaghmaie M, Bashash D, Hassani S, Mousavi SA, Ghaffari SH (2021) Anti-proliferative activity of disulfiram through regulation of the AKT-FOXO axis: A proteomic study of molecular targets. Biochim Biophys Acta Mol Cell Res 1868: 119087. https://doi.org/10.1016/j.bbamcr.2021.119087

Omuro A, DeAngelis LM (2013) Glioblastoma and other malignant gliomas: a clinical review. IAMA 310: 1842-1850. https://doi. org/10.1001/jama.2013.280319

Ostrom QT, Gittleman H, Liao P, Vecchione-Koval T, Wolinsky Y, Kruchko C, Barnholtz-Sloan JS (2017) CBTRUS Statistical Report: Primary brain and other central nervous system tumors diagnosed in the United States in 2010-2014. Neuro Oncol 19: v1-v88. https:// doi.org/10.1093/neuonc/nox158

Pegoraro C, Figueiredo AL, Maczkowiak F, Pouponnot C, Eychène A, Monsoro-Burq AH (2015) PFKFB4 controls embryonic patterning via Akt signalling independently of glycolysis. Nat Commun 6: 5953. https://doi.org/10.1038/ncomms6953

Pilkis SJ, Claus TH, Kurland IJ, Lange AJ (1995) 6-Phosphofructo2-kinase/fructose-2,6-bisphosphatase: a metabolic signaling enzyme. Annu Rev Biochem 64: 799-835. https://doi.org/10.1146/annurev. bi.64.070195.004055

Pranckeviciene A, Bunevicius A (2015) Depression screening in patients with brain tumors: a review. CNS Oncol 4: 71-78. https://doi. org/10.2217/cns.14.60

Ros S, Santos CR, Moco S, Baenke F, Kelly G, Howell M, Zamboni N, Schulze A (2012) Functional metabolic screen identifies 6-phosphofructo-2-kinase/fructose-2,6-biphosphatase 4 as an important regulator of prostate cancer cell survival. Cancer Discov 2: 328-343. https://doi.org/10.1158/2159-8290.CD-11-0234

Rousseau GG, Hue L (1993) Mammalian 6-phosphofructo-2-kinase/ fructose-2,6-bisphosphatase: a bifunctional enzyme that controls glycolysis. Prog Nucleic Acid Res Mol Biol 45: 99-127. https://doi. org/10.1016/s0079-6603 (08)60868-5

Shen C, Ding L, Mo H, Liu R, Xu Q, Tu K (2021) Long noncoding RNA FIRRE contributes to the proliferation and glycolysis of hepatocellular carcinoma cells by enhancing PFKFB4 expression. I Cancer 12: 4099_4108. https://doi.org/10.7150/jca.58097

Strohecker AM, Joshi S, Possemato R, Abraham RT, Sabatini DM, White E (2015) Identification of 6-phosphofructo-2-kinase/fructose-2,6-bisphosphatase as a novel autophagy regulator by high content shRNA screening. Oncogene 34: 5662-5676. https://doi. org/10.1038/onc. 2015.23

Valera ET, Lucio-Eterovic AK, Neder L, Scrideli CA, Machado HR, Carlotti-Junior CG, Queiroz RG, Motta FJ, Tone LG (2007) Quantitative PCR analysis of the expression profile of genes related to multiple drug resistance in tumors of the central nervous system. J Neurooncol 85: 1-10. https://doi.org/10.1007/s11060-007-9382-7

Wang G, Li S, Xue K, Dong S (2020) PFKFB4 is critical for the survival of acute monocytic leukemia cells. Biochem Biophys Res Commun 526: 978-985. https://doi.org/10.1016/j.bbrc.2020.03.174

Yalcin A, Telang S, Clem B, Chesney J (2009) Regulation of glucose metabolism by 6-phosphofructo-2-kinase/fructose-2,6-bisphosphatases in cancer. Exp Mol Pathol 86: 174-179. https://doi. org/10.1016/j.yexmp.2009.01.003

Yan F, Liu R, Zhuang X, Li R, Shi H, Gao X (2021) Salidroside attenuates doxorubicin-induced cardiac dysfunction partially through activation of QKI/FoxO1 pathway. J Cardiovasc Transl Res 14: 355-364. https://doi.org/10.1007/s12265-020-10056-X

Yi M, Ban Y, Tan Y, Xiong W, Li G, Xiang B (2019) 6-Phosphofructo-2-kinase/fructose-2,6-biphosphatase 3 and 4: A pair of valves for fine-tuning of glucose metabolism in human cancer. Mol Metab 20: 1-13. https://doi.org/10.1016/j.molmet.2018.11.013

Yun SJ, Jo SW, Ha YS, Lee OJ, Kim WT, Kim YJ, Lee SC, Kim WJ (2012) PFKFB4 as a prognostic marker in non-muscle-invasive bladder cancer. Urol Oncol 30: 893-899. https://doi.org/10.1016/j. urolonc.2010.08.018

Zhang P, Tao W, Lu C, Fan L, Jiang Q, Yang C, Shang E, Cheng H, Che C, Duan J, Zhao M (2021) Bruceine A induces cell growth inhibition and apoptosis through PFKFB4/GSK3 $\beta$ signaling in pancreatic cancer. Pharmacol Res 169: 105658. https://doi.org/10.1016/j. phrs.2021.105658

Zhang X, Tang N, Hadden TJ, Rishi AK (2011) Akt, FoxO and regulation of apoptosis. Biochim Biophys Acta 1813: 1978-1986. https:// doi.org/10.1016/j.bbamcr.2011.03.010

Zhao Z, Li C, Xi H, Gao Y, Xu D (2015) Curcumin induces apoptosis in pancreatic cancer cells through the induction of forkhead box $\mathrm{O} 1$ and inhibition of the PI3K/Akt pathway. Mol Med Report 12: 5415-5422. https://doi.org/10.3892/mmr.2015.4060 